\title{
The Research of Wire Grinding Machine and Wire Stripping Machine Control System Based On PLC
}

\author{
Lou Feiyan \\ College of Education and Science Technology, Zhejiang University of technology, \\ Hangzhou 310014, China
}

\begin{abstract}
This paper introduces PLC control technology and discusses its characteristics with relay control circuit, computer, SCM control technology then it talks about the PLC control system design methods. To use the method in reality, the paper introduces wire grinding machine and wire stripping machine. In the wire grinding machine, the module is single and easier, with principle of how it works, PLC obtains the status of sensors, data such as the diameter value to control the operation of the corresponding parts to form a closed loop between PLC control system, the motor and wire diameter grinding detectors to grind the wire into segments with different diameters. Finally, the paper completes the PLC-based control system design of stripping machine. The system requires nine different ways to meet the processing requirements, the papers designs multifunctional modules to realize the requirements. Both of the two PLC systems operates very well in reality.
\end{abstract}

Keywords: PLC control system, wire grinding machine, wire stripping machine

\section{Introduction}

As microelectronic technology, control technology and information technology develops, PLC (Programmable Logic Controller) control technology has also developed rapidly [7]. PLC has function such as floating point operation, function operation, highspeed counting, interrupt counting, and PID control and networking, it can be used for industrial control [6].

In the PLC programming language, Ladder is the most used language. PLC ladder diagram is quite similar with relay control circuit, so as input / output of signals and control function, but PLC control and relay control have some differences, mainly in the following aspects. (1) Control logic: relay control logic uses hardwired logic, it uses series contact or parallel contact and time delay relay contact and other combinations of mechanical relay to conduct control logic. The PLC uses its internal memory to store the control logic as program, so it is easy to change the program to change the control logic. (2) Speed control: the relay control action is achieved through mechanical relay contacts, the opening and closing operation of the contacts is usually in the tens of milliseconds [5]. The PLC control logic is achieved by semiconductor circuits through program instructions; the general instruction execution is in microseconds. (3) Limit control: relay control logic uses the time relay to control time, with low timing accuracy, environmental sensitivity, and difficult adjustment. PLC semiconductor is integrated with circuit timer to obtain high timing accuracy, range from $0.001 \mathrm{~s}$ to several minutes, which could be used for timing control through the preparation process, it is very convenient.(4) Count control: Relay control logic generally do not have counting function, but PLC program do have counting function.

PLC is specifically designed for industrial control environment, and general computer is designed for scientific computing and data processing, they both use computer 
architecture, but the difference is mainly reflected in the following aspects [3]. (1) Application: In addition to the field of control, general computer applies in scientific computing, data processing, computer communication and so on, while PLC is mainly used for industrial control. (2) Environment: general computer requires highly for environment, while PLC can be applied to industrial site with poor environment. (3) Programming: general computer has enormous programming languages, such as assembly language, $\mathrm{C}$ language, etc., to achieve complex applications, with high requirement for programmers, while PLC provide little logic programming languages, which are simple, easy to learn and use. (4) Computing speed and storage capacity: the generic computing speed of PLC is relatively slower [10-13].

PLC has a rich instruction set, there are a variety of I/O interfaces[8], communication interface, a large amount of memory, a reliable self-monitoring system, which has the following basic functions: the logic processing functions; data computing capabilities; accurate timing function;-speed counting function; interrupt handling (you can achieve a variety of internal and external interrupt) function[1]; program and data storage capabilities; networking communication function; self-test, self-diagnostic function.

SCM control technology is generally used for data acquisition and industrial control, configuration of microcontroller is simpler than general computer, microcontroller programming is complex and difficult, microcontroller needs to deal with a large number of I/O interface, the output drive load capacity is weak, when driving industrial loads it needs complex peripheral circuits [4]. MCU control technology highlights the advantages that it has strong data processing capacity, but its reliability is far behind PLC in the industrial control process. General SCM control technology is only used in relatively simple industrial process control and occasions where data processing capabilities requirement is relatively high. When PLC control technology is compared to MCU control technology, it is more suitable for process control in industrial field, but its data processing capability is weaker than the latter.

\section{PLC Control System Design Methods}

\subsection{Structure and Working Principle of PLC}

PLC is a core part of the PLC-based control system, PLC and computer are very similar, mainly include central processing unit (CPU), memory, input / output interface, power supply and other components, shown in Figure 1.

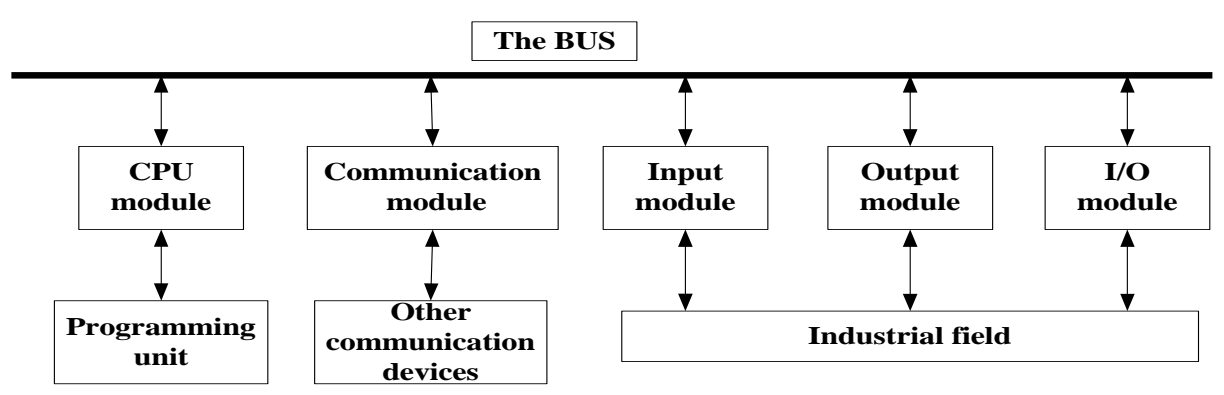

Figure 1. Constitution of PLC

PLC has two operating modes: RUN mode and STOP mode. In the RUN mode, executing user program can be used to fulfill control functions; in STOP mode, CPU does not execute any user program, it can create and edit user program through programming software and download the user program to the PLC. When the PLC is in operation, its work process is generally divided into three stages, namely the input sample, user 
program execution and output refresh. Completion of the three phases is called a scan cycle. During the entire run, CPU of PLC repeat the above three stages with a scanning speed.

\subsection{Design of PLC Control System}

PLC control system consist of hardware and software:(1) The hardware PLC control system should not only meet the requirements of the PLC control system model, memory capacity, input / output module, power module, communication module, analog input / output module, and other special function modules but also meet the requirements of suitable peripheral devices such as input devices (buttons, switches, sensors, etc.), output devices (contactors, relays, etc.) and perform device control field devices (motors, pumps, valves, etc.). (2) PLC control system software includes the use and allocation of I/O addresses, internal relays, timers, counters, PLC and HMI control procedures designed according to the requirements and so on.

The general steps for the PLC control system design:

(1) Control requirements analysis: Before designing the PLC control system, it is important to understand and analyze the controlled object process requirements and control requirements to design a satisfactory control system.

(2) Determine input / output device: according to the control requirements, select the appropriate input device (control buttons, switches, sensors, etc.) and output devices (contactors, relays, etc.), and determine the required PLC I/O interfaces.

(3) Select the appropriate PLC: according to the desired I/O interfaces and specific PLC control system requirements, with consideration of PLC models, storage capacity, power supply modules and other modules.

(4) Assign I/O: decide the correspondence between I/O terminals and I/O devices.

(5) PLC programming: make PLC programming based on the control object and control requirements.

(6) Simulation debugging: You can use the buttons, switches to simulate digital, voltage and current sources instead of analog, repeat testing program, then modify until it meet the control requirements.

(7) On-site installation: connect the input / output devices and PLC.

(8) Online debugging.

(9) Finish Technical Documentation.

\section{Design and Implementation of Single-Function Module for Grinding Machine Control System}

\subsection{Grinding Machine System}

There are strict requirements on the flatness of end surface for special wire in aviation, aerospace, defense industry and other military fields. However, when cutting wire, it is easy to produce squeeze on core wire and make damage on the end. Therefore it is need to polish core wire end surface. Traditional industrial grinding machine is currently used in the production line, but it can only remove insulating paint and other surface impurities.

The device uses three servo motors to drive belt pair to move relatively from the wire, so that the belt surface and the end of the wire can contact all round to achieve the purpose of grinding wires. Among them, the lateral motor control feeding belt, change the distance between the belt and the core wire end surface; longitudinal motor control belt 
move left and right to achieve frictional move between belt and wire core; vertical motor control rotation of belt. In order to ensure full cross-section of the contact wire core end and belt, and improve the efficiency of grinding wire, during the belt longitudinal movement, lateral motor slowly drives belt to feed forward. Vertical motor rotates after each longitudinal movement to improve the tape utilization.

Grinding Machine system is consist of core controller, diameter detection system, wire transfer system, grinding wire servo system, packing wire servo system. Figure2 shows the structure diagram of grinding wire system.

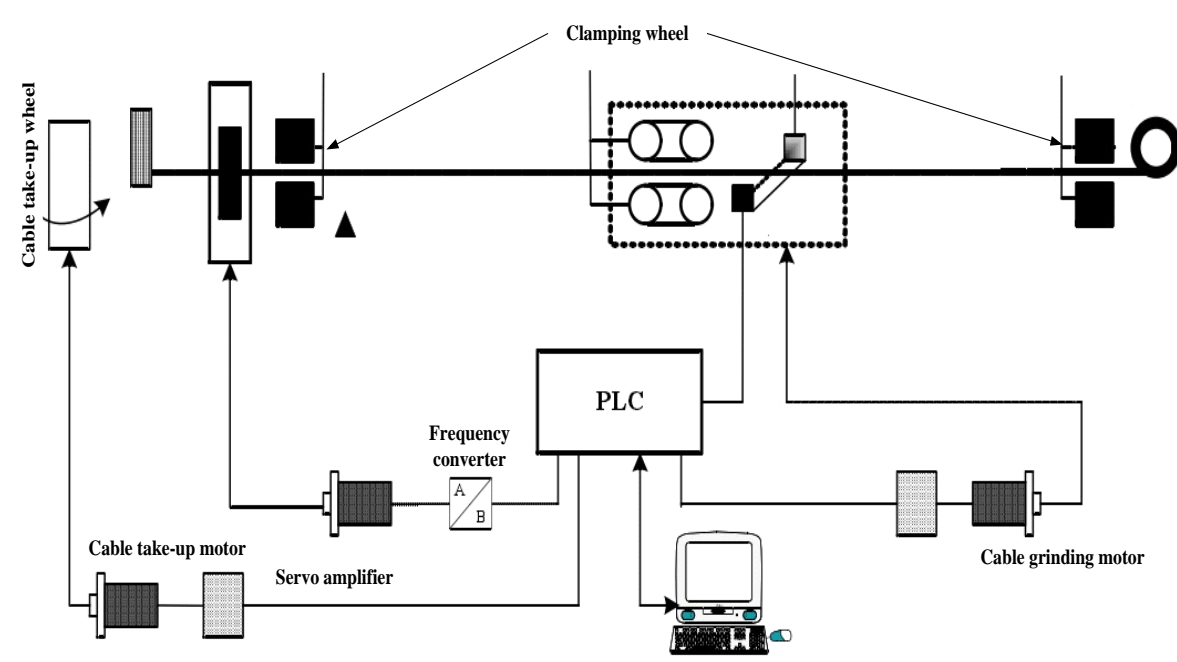

Figure 2. The Structure Diagram of Grinding Wire System

Lateral motor moving back and orientation, vertical motor moving back, and vertical motors progressing, are all single-step movement, the paper uses match interrupt to control. Horizontal, vertical motors process in parallel, the progressive distance ratio between the lateral and vertical progression at any time should be equal to the total distance ratio. If you are using two interrupt commands to transmit pulse, interference occurs between the interrupt commands, it is difficult to coordinate and control horizontal and vertical feed distance, thus completing the parallel control in a match interrupt. Since the maximum distance of the vertical motor is $90 \mathrm{~mm}$, while the progressive lateral distance of each motor does not exceed $0.2 \mathrm{~mm}$, the number of horizontal pulses is much larger than that of vertical pulses, so the vertical movement of the motor is a benchmark in the linkage process. Meanwhile, according to the ratio between the number of horizontal and vertical pulses the pulse, when the motor produces certain amount of pulses for the longitudinal direction, a pulse is sent to the motor. For example, it is need to send 4000 longitudinal direction pulses and 125 horizontal pulses, it means sending 16 longitudinal pulses per horizontal pulse. This control method ensures real-time progressive vertical and horizontal length is not affected by changes of the speed of longitudinal motor.

Grinding machine system has three modes: manual mode, automatic mode and packing wire mode, which could be switched through PC's user interface.

(1) Manual mode: the control system drives spinning wire system to complete the installation. The thin metal wire starts from wire pack, after passing through rear gripping wheel, bench, front gripping wheel, it is pulled tightly by panel to complete assembly wire. Under manual mode, the following actions can be controlled separately, such as clamping and gripping and rotating wheels, table moving forward and backward, belt clamping down and relax, belt rotation and tension plate stretching.

(2) Automatic mode: the control system mainly drives grinding system to complete grinding thin metal wire. First, wire motor will turn grinding table to the left to the 
starting point, to make sure the front and rear clamping wheels locked, and then start spinning wire motor, so that the thin metal wire rotates at high speed. When starting grinding, the upper and lower belt holds thin metal, then table moves, the belt could grind metal wires. In this mode, PLC calculates various data real-time, such as the wire length of each grinding and each grinding diameter, then according to the calculating results and the real-time detected value of diameter, the grinding wire machine could automatically complete the entire grinding task.

(3) Packing wire mode: the control system drives packing wire system to complete the packing wire. After relaxing the tension plate and front and rear clamping wheel, the head of wire grinded would be fixed at packing wire wheel, then the motor starts packing wire as set beforehand.

Figure3 shows the schematic diagram of closed loop control system, which is composed by PLC, motor and grinding wire diameter detector. PLC control system controls the grinding machine to polish metal thin wire, after completing a task, the diameter detector feeds back current diameter value, if the current value is less than the diameter of the last part diameter value, the control motor starts grinding the next part wire; otherwise, polishes the current part again.

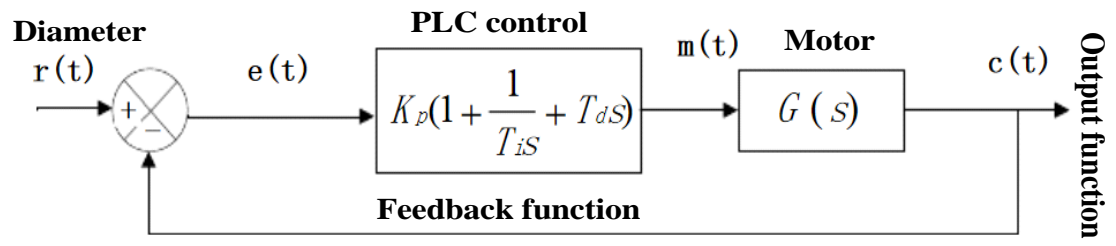

Figure 3. Diagram of Closed Loop Control System

\subsection{Hardware Design of Grinding Machine Control System}

When selecting the PLC, the following factors should be considered: machine model, capacity, I / O module, power module. In wire grinding machine control system, after research and analysis, the paper chose Mitsubishi FX2N series as the system controller of PLC. Mitsubishi FX2N series is the most advanced super-micro PLC FX series, in addition to the general input-output function, it has special functions such as analog control and positioning control.

Considering the PLC cannot directly use output analog signal of diameter detector, the paper uses Mitsubishi FX2N-2AD analog input module to change the analog signal to a digital signal.

Considering the PLC cannot directly use output analog signal of diameter detector, the paper uses Mitsubishi FX2N-2AD analog input module to change the analog signal to a digital signal.

In wire grinding machine control system design, the paper chooses Mitsubishi MR-E$200 \mathrm{~A}$ as servo amplifier of grinding wire system, MR-E-100A as servo amplifier of closing wire system. Universal AC Servo Mitsubishi MR-E series has position control and speed control modes. MR-E series servo motor encoder with 10,000 pulses/rev resolution incremental position encoders, can be high-density positioning.

Command pulse using the timer interrupt matching output, thus realize position control, equation is expressed as:

$$
\text { Speed }=\frac{\text { Telock }}{\text { Rate }}
$$


In the equation, Speed is the speed of motor, the unit is PPS; Telock is the clock of parameters, it can choose the $1 \mathrm{MHz}, 1 / 4 \mathrm{MHz}, 1 / 16 \mathrm{MHz}$. Rate is the pulse rate. We can get the linear relation from the equation, as the figure 4 shown.

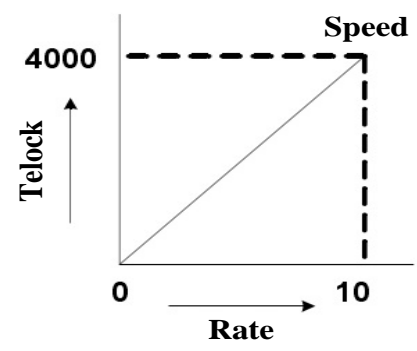

Figure 4. Command Pulse

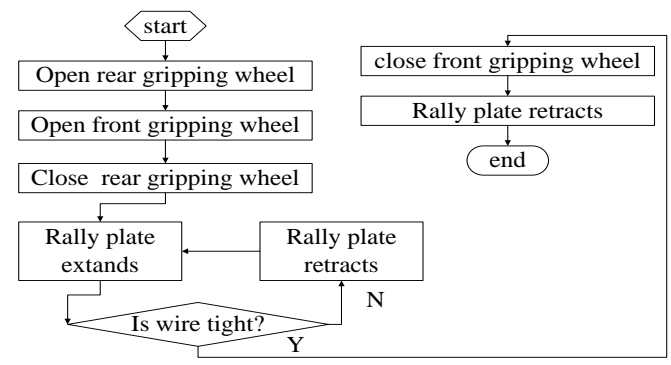

Figure 5. The Logic Diagram of Assembly Wire

\subsection{Software Design of Grinding Machine Control System}

According to the design requirements, software design grinding wire control system includes the PLC program design and human-computer interface design.

After detailed study working principle and control requirements of grinding machine, the system uses modular design ideas, which consists of four parts: assembly wire modules, grinding wire module, the spinning wire module, wire diameter sampling module. Writing programs uses ladder programming, key module, automatic grinding wire module, uses stepping sequence instructions method.

Assembly wire module is responsible for automatic grinding preparatory work of wire module, assembly wire module requires to lead thin metal wire from the right of grinding machine, then through the front holding wheel and back holding wheel, pull the wire to the proper tightness with tension plate, all the actions are finished through the manmachine interface control step by step manually. Figure5 shows the logic module assembly wire process.

Automatic grinding wire module is the core part of the grinding wire control system. Considering the automatic grinding wire module is executed by the order, so when programming this part, the paper uses Mitsubishi GX developer software controlling step instructions to control every step of the action, the end of the previous step is trigger conditions of the next step. After detailed research and analysis, the paper designs a sequential function chart of automatic grinding wire module.

Spinning wire module is to spin the polished the metal wire around the spinning wheel. The end of one action of spinning wire is the begin of next action, each action must be done step by step, all actions are also controlled by the man-machine interface manually completed. There are many interlocking flags in the spinning wire module to ensure that the next manual control action to be effective after the previous step is completed. Figure6 shows the process of spinning wire. 
The design idea of wire diameter sampling module: take twenty numbers at per sampling point, remove the maximum and the minimum, and then take the average value of the remain as the sampling point, also as the real-diameter value; each take a sampling point each three $\mathrm{cm}$, finally take averaged data of five sampling points as the ultimate value of sample diameter. Figure7 shows the process of wire diameter detecting.

In this system, the paper uses sub-control technology developing king view to design human-computer interface. Configuration software is a software platform for the monitoring and data collection, it can provide a good user interface and is simple to use development method with flexible configuration mode (rather than programmatically).

The grinding machine control system has following superiorities:

(1) Simple structure, convenient operation, high reliability.

(2) Good quality of grinding wire. End surface of core wire is polished smooth; closedloop control of the motor makes grinding wire error is less than $0.1 \mathrm{~mm}$.

(3) Strong adaptability. Device set grinding wire length, the number of parameters to accommodate various materials, diameters, to meet the different requirements of grinding length. The device has been put into use, and achieved good results. For $1 \mathrm{~mm}$ diameter copper wire, it can grind $1 \mathrm{~mm}$ length and obtain smooth end surface no more than $10 \mathrm{~s}$; grinding wire error is less than $0.1 \mathrm{~mm}$.

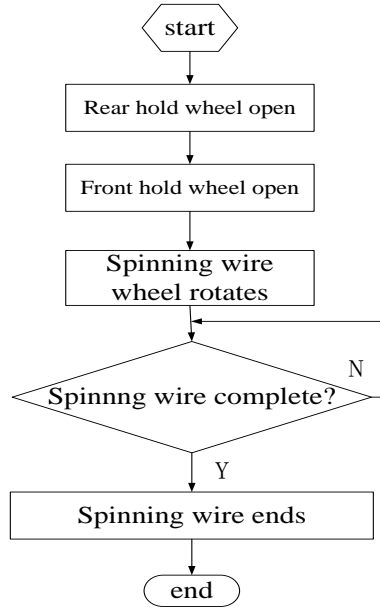

Figure 6. The Process of Spinning Wire

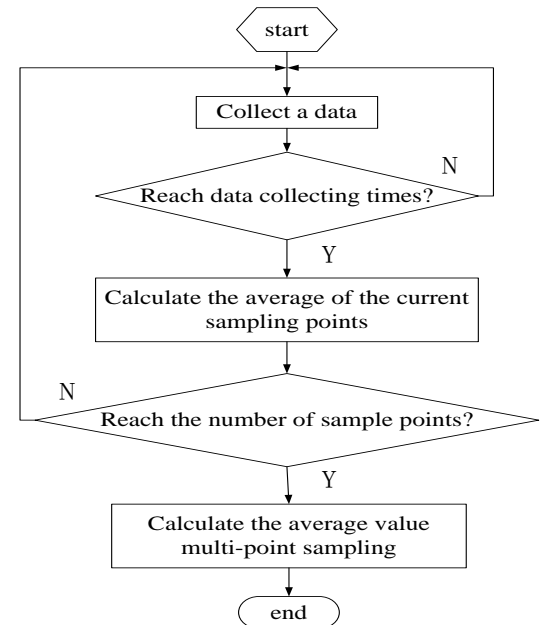

Figure 7. The Process of Wire Diameter Detecting 


\section{Design and Implementation of Multifunctional Module for Stripping Machine Control System}

\subsection{Stripping Machine Control System}

In recent years, domestic and foreign manufacturers have designed a series of wire strippers, such as the small temple C351 stripping machine in Japan, which uses the special stepper motor, and is full-featured, small sized, light weighted, but the price is too high for users to accept; American ARTOS machine which uses pneumatic valve servo system, with big power, three cutters work simultaneously, stripping speed is fast, but the accuracy is poor, the volume is big, noise is loud, and the machine is difficult to adjust. In 1980s there are some of the early manufacturers of generic Japan products in China. In early 1990s, domestic stripping machine started production. With the development of electronic technology, in late 1990s, stripping machine uses a common stepper motor, reducing product cost, there is a good market prospects.

Wire stripping process can be divided into the following steps: sending thread (tail), fixing wire, stripping head (tail), releasing wire, delivery wire length, cutting wire, wire feeding. Figure 8 shows stripping machine system structure, stripping machine system mainly consists of three parts, namely LCD, PLC control, stepper motor. LCD put information into microcontroller and PLC keyboard to control microcontroller and LCD screen; PLC acts as required to programming to control stepper motor to complete system functions, some error messages would be sent by the PLC code to microcontroller to display on the screen after decoding; stepper motor includes four stepper motors and drives, stepper motor drive receives pulses sent by PLC and direction signals to drive the motor control the corresponding left wheel, cutter, right wheel and twisted wire wheel movement.

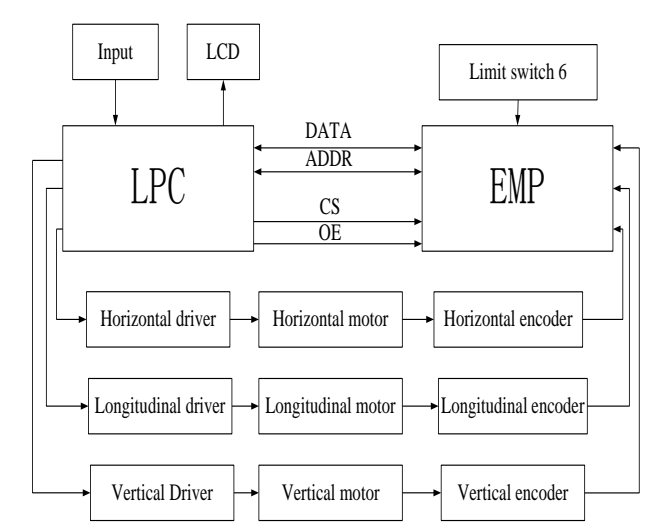

Figure 8. Stripping Machine System Structure

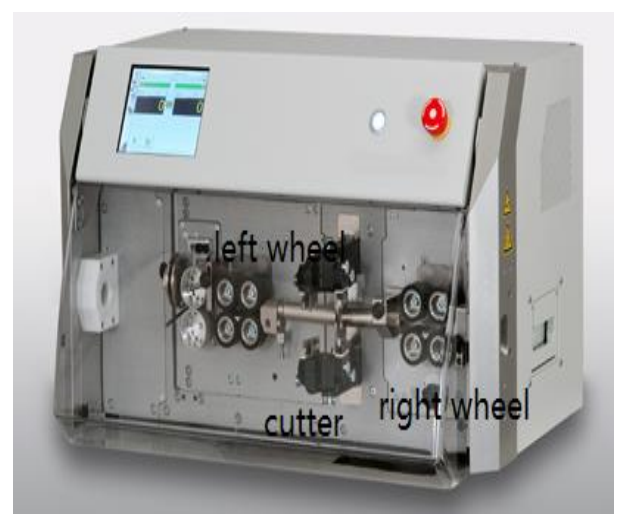

Figure 9. Stripping Machine System Working Principle 
Figure9 shows stripping machine system working principle, the right stepper motor drives the above and below wheel to rotate, moves around the clamped processing line; the cutter stepper motor drives the above and below two cutter in the opposite direction, through controlling of the displacement between the above and below cutter to realize the function of peeling and cutting; left stepper motor drives above and below wheel on the left to rotate, thereby stimulating clamped processing line and move around; the twisted wire stepper motor drives the left wheels in the opposite direction to achieve twisting wire function.

Specific design requirements of stripping machine: set parameters (according to different initial settings, stripping machine has nine different ways of working), start and stop control, continuous stripping, automatic counting, real-time display, error warning. The wire stripping machine working types show in the Table.1.

Table 1. Wire Stripping Machine Working Types

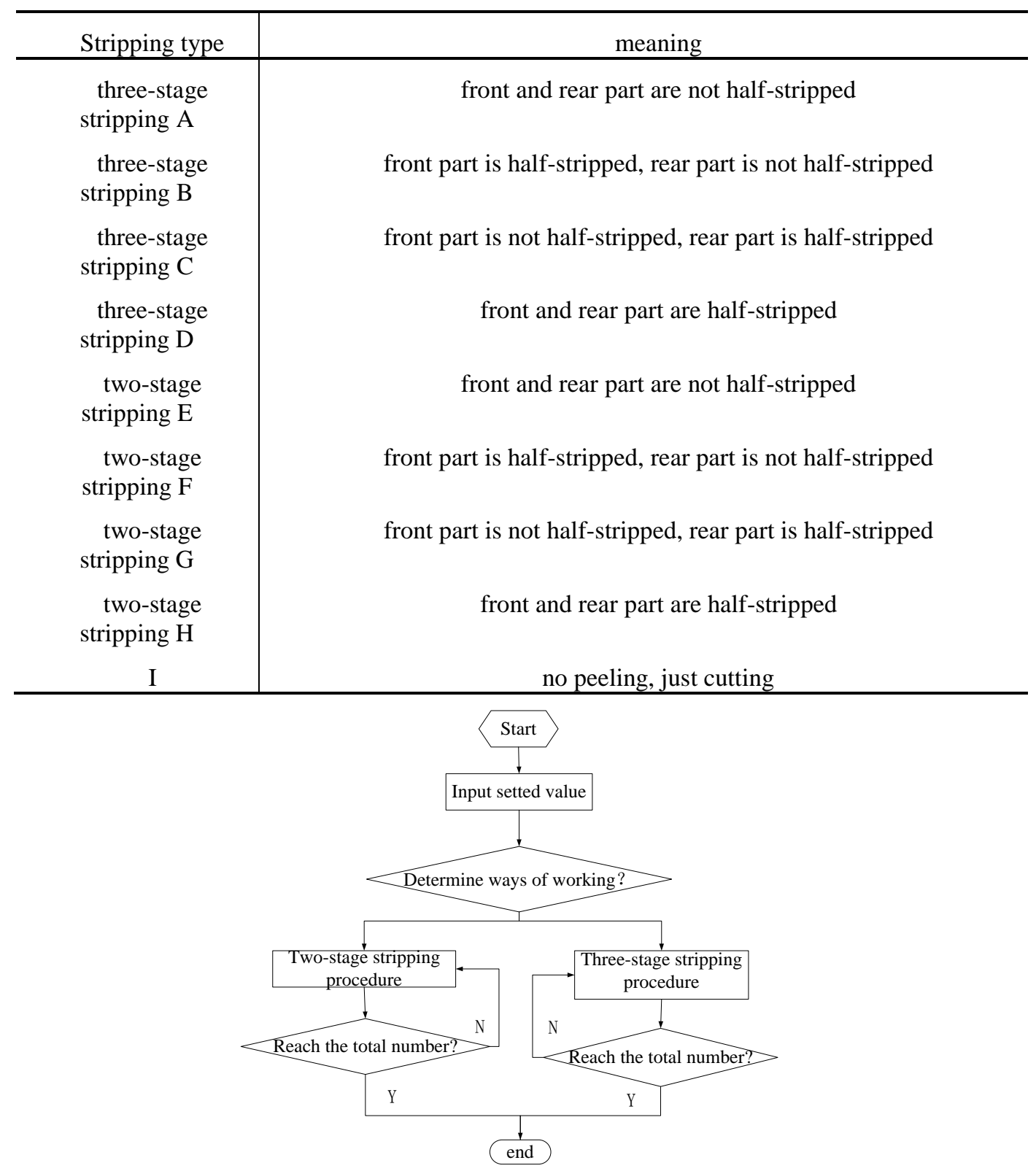

Figure 10. Main Program of Stripping Machine Control System 


\subsection{Hardware Design and Software Design of Stripping Machine Control System}

According to the design requirements, stripping machine control system has 36 input buttons and three sensors (namely knife homing sensor, twisting wheel homing sensor and wire detection sensor), which means there are 39 inputs in total.

Stripping machine control system is consist of nine inputs, namely PLC needs 9-point inputs; output of PLC mainly controls four stepper motors and error information coding, which means 16-point output was needed.

With comparison and analysis, the paper chose Panasonic FP0-T32 type PLC. Panasonic FP0-T32 not only has the general input and output function, but also has a very rich expansion unit, such as a wide selection of I/O point extensions, which can easily achieve extended function. Figure 11 shows the PLC input and output wiring diagram of stripping machine control system. Figure10 shows the main program of stripping machine control system, Figure12 shows the program of two-stage stripping, the program of threestage stripping is almost the same as two-stage stripping.

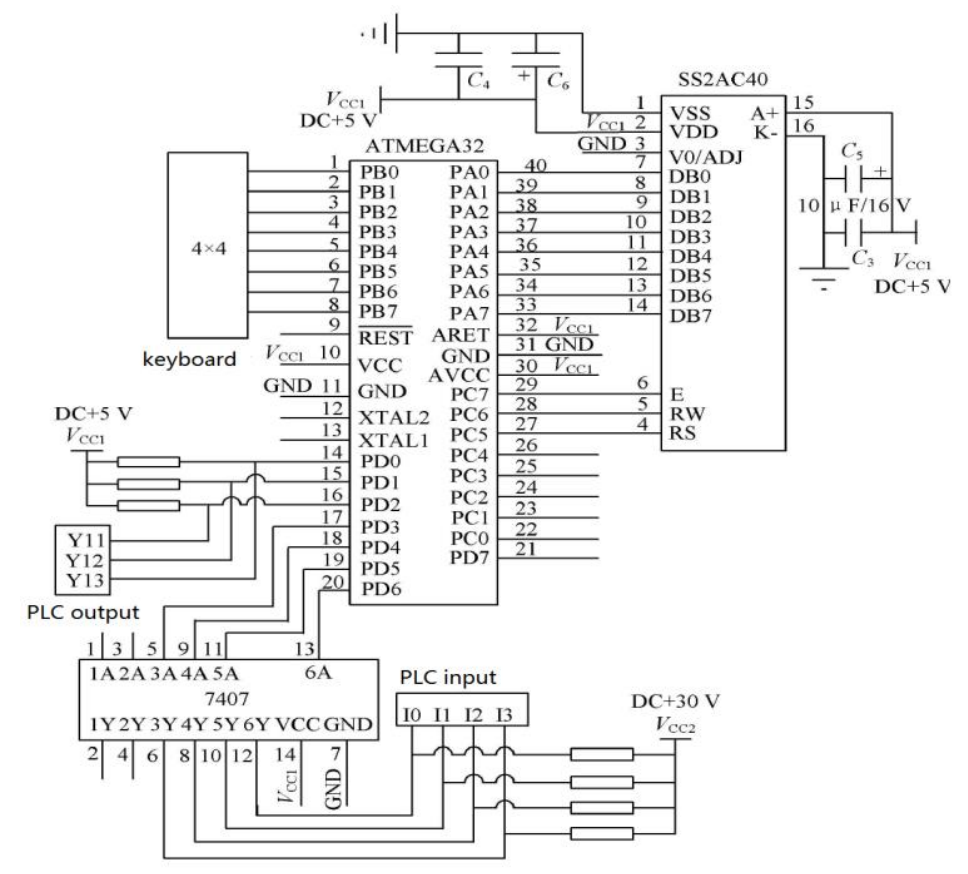

Figure 11. Plc Input and Output Wiring Diagram of Stripping Machine Control System 


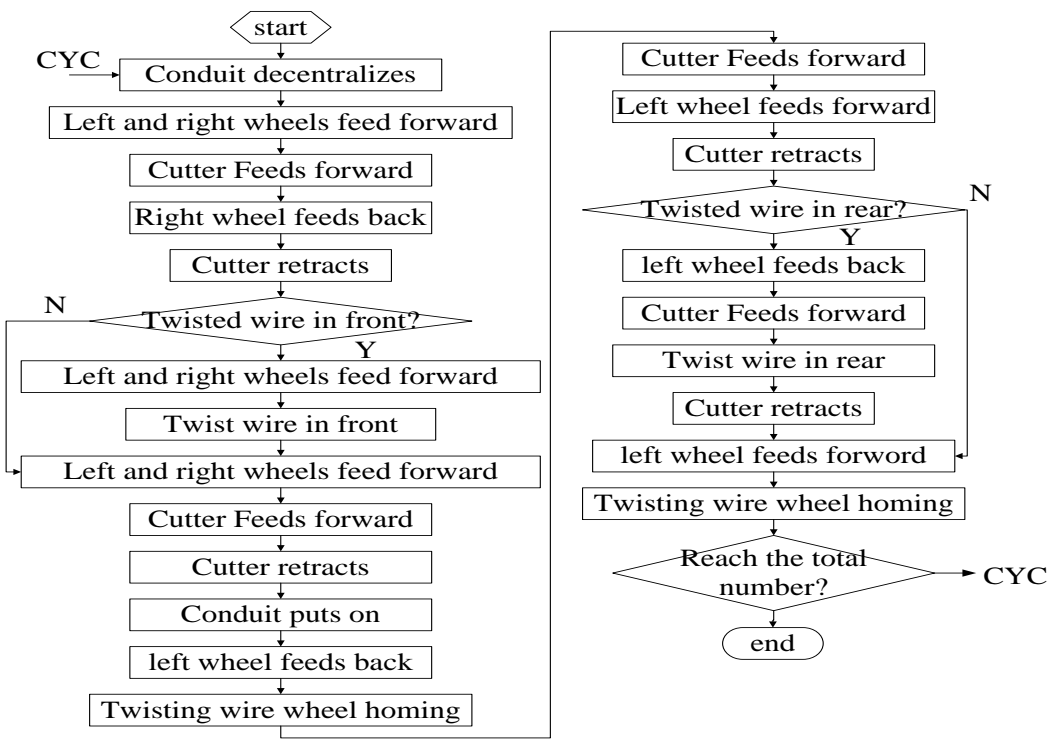

Figure 12. Program of Two-Stage Stripping

The product has an automatic feeding wire, automatic qualifying, unique stripping placing, timing, no wire knotted automatic shutdown, ultra short-stripping, coaxial cable and sheath stripping functions. Product specifications are shown in Table 2. In addition, the mechanical structure adds the drive mechanism to realize thread tail twisted wire, cable stripping and other functions. In the process of stripping, the product has a good effect of stripping.

Table 2. Wire Stripping Machine Specifications

\begin{tabular}{c|c}
\hline Grinding wire length & $1 \mathrm{~mm} \sim 9999 \mathrm{~mm}$ \\
\hline Grinding length & Head $0.12 \mathrm{~mm} \sim 25 \mathrm{~mm}$, tail $0.12 \mathrm{~mm} \sim 15 \mathrm{~mm}$ \\
\hline Wire core & $0.1 \sim 4$ AWG 10\# 32\# \\
\hline error & $\leq 0.002 \times \mathrm{L} \mathrm{mm}$ \\
\hline Stripping place & 9 \\
\hline Wire Pressure regulator & have \\
\hline Pulse equivalent & No error in $1 \mathrm{~m}$ \\
\hline
\end{tabular}

\section{Conclusion}

This paper uses grinding wire machine control system and stripping wire machine control system as the object, from the perspective of PLC -based control system design to explore PLC control technology. In order to grind uniform, non- tapered metal wire as the requirements, there is processing method polishing wire into multiple segments with different diameter. In this system, PLC obtains the status of sensors, data such as the diameter value to control the operation of the corresponding parts of other machinery to form a closed loop between PLC control system, the motor and wire diameter grinding detectors. Finally, to complete the PLC-based control system design of stripping machine. The system requires nine different ways to meet the processing requirements, the difficulty lies in the design of high-speed pulse emitted by PLC precise control of the four stepper motors. In hardware design, to save costs, 36 input keys uses matrix-encoded 
converted into six -way PLC inputs. To prevent the loss of stepper motor step and improve stability and reliability of the stepper motor, the paper uses a ladder-shaped acceleration and deceleration curve control method, with modular design of the PLC program, to achieve precise control of the stepper motor.

\title{
References
}

[1] P.Cleaveland, Programmable Controllers Adapt to New Industry Needs, Control Solutions, vol.4, no.1, pp. 47-50(2001).

[2] D. W. Russell, Application of PLC's as front-end pre-processors in factory information systems, The International Journal of Advanced Manufacturing Technology, vol.6, no. 4, pp. 364-377(1999).

[3] Frederico de Araujo Kronemberger and Lidia Maria Melo Santa Anna, Oxygen-controlled Biosurfactan Production in a Bench Scale Bioreactor, Applied Biochemistry and Biotechnology, vol.19, no.6, pp.201203(2007).

[4] Atef A. Ata, Ahmad Rafeekand and Hanani Yusof, Sensory-Based Colour Sorting Automated Robotic Cell, Journal of Intelligent and Robotic Systems, vol.41, no.1, pp. 99-110(2005).

[5] Hu W.Schroeder and M.Starr A.G, A knowledge-based real-time diagnostic system for PLC controlled manufacturing systems, IEEE SMC 99 Conference Proceeding, vol.4, no.31, pp. 499-504(1999).

[6] Frey G and Litz L, Formal methods in PLC programming, IEEE International Conference, vol.4, no.6, pp. 2431-2436(2000).

[7] Ioannides, M.G, Design and implementation of PLC-based monitoring control system for induction Motor, IEEE Transaction on Energy Conversion, vol.19,no.3, pp.469 - 476(2004).

[8] GangLi and JingYing, A Configuration Software System for Industrial Monitoring and Controlling". Proceedings International Conference, vol.6, no.6, pp. 466-470(2004).

[9] D. O. Carrica, S. A. González and M. Benedetti, A high speed velocity control algorithm of multiple stepper motors, Mechatronics, vol.14, no.6, pp. 675-684(2004).

[10] I.M.EI-Amin, A.R.AI-Ali and M.A.Shuail, Direct Load Control Using a Programmable Logic Controller". Electric Power Systems Research, vol.52, no.3, pp. 211-216(1999).

[11] Ball K, PLC I/O systems news, views, and networks, Control Engineering, vol.44, no.14, pp. 8188(1997).

[12] Nanette Bauer, Ralf Huuck, Ben Lukoschus and Sebastian Engell, A Unifying Semantics for Sequential Function Charts". Control Engineering, vol.4, no.7, pp. 51-58(1999).

[13] Frey G and Litz L., Formal methods in PLC programming, IEEE International Conference, vol.4, no.7, pp. 2431-2436(2000).

\begin{abstract}
Author
Lou Feiyan, was born in 1976 at Zhejiang hangzhou, and receive $\mathrm{PhD}$ of mechatronic engineering from the "Zhejiang University of Technology" in 2009. She is now a teacher of the College of Education and Science Technology, Zhejiang University of Technology. Her research interests include the design and manufacturing of mechanical equipment, electrical control and PLC application etc.s
\end{abstract}

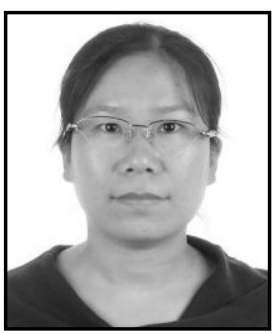

